\title{
A Retrospective Analysis of the Significance of Serum Bilirubin Levels and Glycemic Measurements in Type 2 Diabetes Management in a Cohort of Patients in a Tertiary Care Hospital
}

Chitranga Chysanthi Kariyawasan

Sri Jayewardenepura General Hospital

Chitranga Chysanthi Kariyawasan ( $\square$ Kchysanthi@gmail.com )

Sri Jayewardenepura General Hospital

Balasuriya Lekamlage Thushara Balasuriya

Sri Jayewardenepura General Hospital

Sondapperuma Arachchige Charith Dakshitha Ranatunga

Sri Jayewardenepura General Hospital

Dissanayake Mudiyanselage Champika Dissanayake

Sri Jayewardenepura General Hospital

Singappuli Ralalage Gemunu Priyantha Herath

Sri Jayewardenepura General Hospital

Research article

Keywords: Type 2 Diabetes mellitus(T2DM), Serum Bilirubin, antioxidant, oxidative stress, Glycated Haemoglobin (HbA1c), oxidative stress-mediated disease, fasting blood sugar(FBS), post prandial blood sugar(PPBS), advanced glycation end products (AGE)

Posted Date: October 5th, 2021

DOl: https://doi.org/10.21203/rs.3.rs-955361/v1

License: (a) This work is licensed under a Creative Commons Attribution 4.0 International License. Read Full License 


\section{Abstract}

\section{Background}

In type 2 Diabetes Mellitus (T2DM) chronic hyperglycemia leads to multi-organ damage. It is a chronic low-grade inflammation resulting in reduced antioxidants leading to many complications. Hyperglycemia causes glycation of serum proteins forming advanced glycation end products (AGEs). HBA1c is an AGE of haemoglobin. Bilirubin considered a waste product of the heme catabolic pathway, has proven to be a natural antioxidant associated with a lower prevalence of oxidative stress-mediated diseases. Bilirubin prevents glycation of proteins providing benefit in T2DM.

General objective - To evaluate the association of serum bilirubin levels with HBA1c, FBS, PPBS in diabetes patients.

Specific objectives - 1) To compare the serum bilirubin levels in diabetes patients with good and poor control. 2) To evaluate the association of $\mathrm{HbA} 1 \mathrm{c}$ with FBS, PPBS and age in controlled and uncontrolled diabetes mellitus

Methodology -A retrospective analytical study done at the department of Haematology of the Sri Jayewardenepura General Hospital.

Sample selection and sample size -A random sampling technique used. Data obtained through laboratory information system of 201 adult patients diagnosed with T2DM. The study population grouped as poorly and well controlled using $\mathrm{HbA} 1 \mathrm{c}$ value over and below $7 \%$ respectively. Normal ranges for serum bilirubin, FBS and PPBS was (0.3-1.2) mg/dl, (75-110) mg/dl and (80-140) $\mathrm{mg} / \mathrm{dl}$ respectively. Data were entered and analyzed using statistical package for social sciences (SPSS) version 20. Descriptive statistics used to calculate the median, mean and the standard deviation of age, serum bilirubin, HBA1c, FBS, and PPBS. Correlations between variables were done using Pearson's Correlation method. The $p$ value $<0.05$ was considered as statistically significant. Comparison of two diabetic groups were done using independent sample t test.

Results. A negative correlation between total bilirubin and $\mathrm{HbA1c}(r=-0.234$, $p$ value $=0.001)$ with a low linear relationship (R Sq of 0.055 , figure 02 ) observed. No statistical significance between total bilirubin level with FBS, PPBS and age ( $p$ value $>0.05$ ) but the mean values of these in the two groups were statistically significant $(p$ value $<0.05)$. A statistically positive co-relation observed between $\mathrm{HbA} 1 \mathrm{c}$ with FBS and PPBS, not with age.

Conclusion. The significant negative correlation between total bilirubin and $\mathrm{HbA} 1 \mathrm{c}$, infers that a lower blood glucose level will result in reducing the oxidant stress mediated damage by the antioxidant and anti- inflammatory action of the increased oxygen free radicals produced by a high bilirubin.

\section{Background}


Type 2 Diabetes Mellitus (T2DM) is an endocrine disorder. This is characterized by high levels of glucose in blood resulting from variable degrees of insulin resistance and deficiency. Chronic hyperglycemia can lead to multi-organ damage resulting in many complications on renal, neurologic, and cardiovascular systems ${ }^{1}$.

The worldwide prevalence of adult T2DM is $9 \%$ in men and $7.9 \%$ in women ${ }^{2}$. In 2005, a study done on adults between 35 and 65 years of age in four provinces (Western, North Central, Southern and Uva) in Sri Lanka, reported prevalence of diabetes as $14.2 \%$ and $13.5 \%$ in men and women respectively ${ }^{3}$.

Glycemic control is very important in reducing morbidity and mortality of the disease. Control of blood glucose levels in patients with diabetes can be determined by estimation of Glycated Hemoglobin (HbA1c), Fasting Blood Sugar (FBS) and Post Prandial Blood Sugar (PPBS). HbA1c level remains the gold standard for assessment of diabetes at follow up ${ }^{4}$.

T2DM is a chronic low-grade inflammation which causes reducing antioxidant capacity of the human body. In T2DM, hyperglycemia is the main cause for the complications. Hyperglycemia causes glycation of serum proteins. In glycation, free amino groups of proteins, lipids and nucleotides are modified by monosaccharides. During the reaction, advanced glycation end products (AGEs) are formed. Chronic hyperglycemia accelerates the reaction between monosaccharides and proteins to form of AGEs. HBA1c is also an AGE that the haemoglobin compound produced after glucose reacts with the amino group on a hemoglobin molecule forming a ketoamine. ${ }^{5}$

HBA1c level indicates the average blood sugar over the past 3 months. Regardless of underlying treatment, $\mathrm{HbA} 1 \mathrm{c}$ more than $7.0 \%$ is associated with a significantly increased risk of both microvascular and macrovascular complications. Every $1 \%$ reduction in $\mathrm{HbA} 1 \mathrm{c}$ is said to result in nearly $20 \%$ decrease in the risk of death related to diabetes and more than $35 \%$ decrease in risk of microvascular complications. ${ }^{6}$

A few decades ago, bilirubin was believed to be only a waste product of the heme catabolic pathway and potentially toxic compound. But recent data has shown that mildly elevated levels of serum bilirubin are strongly associated with a lower prevalence of oxidative stress-mediated diseases. Bilirubin is a natural anti-oxidant of the body which has protective effects against free oxygen radicals. Bilirubin plays an important role in preventing glycation of proteins. This capability is an important benefit in T2DM and macrovascular diseases. ${ }^{7}$

\section{Objectives}

\section{General Objective}

To evaluate the association of serum bilirubin levels and the measurement of HBA1c, FBS, PPBS in diabetes patients.

\section{Specific objectives}


1. To compare the serum bilirubin levels of diabetes patients with good control as opposed to those with poor control.

2. To evaluate the association of HbA1c with FBS, PPBS and age in controlled and uncontrolled diabetes mellitus

\section{Methodology}

\section{A. Study design}

A retrospective analytical study.

\section{B. Center of the study}

Department of Haematology of the Sri Jayewardenepura General Hospital.

\section{Sample selection and sample size}

A simple random sampling technique was used, over a period of 6 months March 2020 to September 2020 , to obtain the laboratory records of 201 adult patients both males and females who were diagnosed with T2DM, through laboratory information system

Investigations of $\mathrm{HbA1c}$, FBS, PPBS and serum bilirubin levels were recorded. The patients diagnosed with the Type 1 Diabetes Mellitus, patients with hypothyroidism, Cushing's syndrome, chronic systemic illness, hepatic impairment, renal disorders, heart failure, pregnancy and cancer were excluded from the study.

Our study population was grouped into two according to the $\mathrm{HbA} 1 \mathrm{c}$ level. Subjects with $\mathrm{HbA} 1 \mathrm{c}$ level over $7 \%$ were grouped as poorly controlled and $7 \%$ or lower were grouped as well controlled T2DM. Normal range for serum bilirubin for adults was (0.3-1.2) $\mathrm{mg} / \mathrm{dl}$. Normal ranges for FBS and PPBS were (75-110) $\mathrm{mg} / \mathrm{dl}$ and (80-140) $\mathrm{mg} / \mathrm{dl}$ respectively.

\section{Data Collection}

Automated chemistry analyzer Mindray BS-480 and Abbott Architect plus analyzer were used for the estimation of FBS and PPBS. Serum bilirubin was estimated by Abbott Architect plus analyzer.

HbA1c was estimated by High-Performance Liquid Chromatography (HPLC) method on BIO RAD D10 analyzer.

\section{E. Statistical Analysis}

Data were double entered and were analyzed using Statistical Package for Social Sciences (SPSS) version 20. Descriptive statistical methods were used to calculate the median, mean and the \pm standard deviation of age, serum bilirubin, HBA1C, FBS, and PPBS. Correlations between study variables were done 
with Pearson's Correlation method. The $\mathrm{p}$ value, lower than 0.05 was considered as statistically significant. Coefficient of determination (R Sq) was used to a statistical measure of how close the data are to the fitted regression line. Two diabetes groups were compared by independent sample $t$ test.

\section{F. Ethical Clearance}

Ethical approval was obtained from the ethical committee of the Sri Jayewardenepura General Hospital.

\section{Results}

Of the total of 201 patients' data was analyzed, 136 were female and 65 were males. Age range was between 31 to 83 years and mean age \pm Standard Deviation (SD) was $59.3 \pm 10.56$ years. The data was categorized under six age categories (Table 01).

Table 01

- Age categories

\begin{tabular}{|ll|}
\hline Age Category (Years) & Frequency \\
\hline $31-40$ & 11 \\
$41-50$ & 32 \\
\hline $51-60$ & 64 \\
$61-70$ & 68 \\
\hline $71-80$ & 23 \\
$81-100$ & 3 \\
\hline
\end{tabular}

The mean \pm SD values of total bilirubin, HbA1c, FBS and PPBS were $0.61 \pm 0.34 \mathrm{mg} / \mathrm{dl}, 7.85 \pm 1.84 \%$, $134.0 \pm 54.6 \mathrm{mg} / \mathrm{dl}$ and $182.7 \pm 83.4 \mathrm{mg} / \mathrm{dl}$ respectively (Table 02 ).

Table 02

- Descriptive data of Age, Total bilirubin, HbA1C, FBS and PPBS.

\begin{tabular}{|llllll|}
\hline Variable & Unit & Minimum & Maximum & Mean & Std. Deviation \\
\hline Age & Years & 31.0 & 83 & 59.32 & 10.56 \\
\hline Total bilirubin & $\mathrm{mg} / \mathrm{dl}$ & 0.2 & 2.6 & 0.61 & 0.34 \\
\hline HbA1C & $\%$ & 4.7 & 14.5 & 7.85 & 1.84 \\
\hline FBS & $\mathrm{mg} / \mathrm{dl}$ & 55.7 & 425.7 & 134.0 & 54.60 \\
\hline PPBS & $\mathrm{mg} / \mathrm{dl}$ & 59 & 486 & 182.7 & 83.44 \\
\hline
\end{tabular}

The mean \pm SD value of total bilirubin was high in the (41-50) age category and it was $0.762 \pm 0.55$ $\mathrm{mg} / \mathrm{dl}$. The age category (51-60) showed the highest mean \pm SD value for HbA1c (8.25 $\pm 2 \%)$. But (61- 
70) age category and (81-100) age category showed the highest mean value of FBS $(140.6 \pm 62.4$ $\mathrm{mg} / \mathrm{dl})$ and PPBS (233.1 $\pm 103.1 \mathrm{mg} / \mathrm{dl}$ ) respectively (Table 03$)$.

Table 03

- Descriptive data for age categories

\begin{tabular}{|c|c|c|c|c|c|}
\hline Variable & Age Category (Year) & Minimum & Maximum & Mean & Std. Deviation \\
\hline \multirow[t]{6}{*}{ Total Bilirubin } & $30-40$ & 0.3 & 0.7 & 0.49 & 0.15 \\
\hline & $41-50$ & 0.3 & 2.6 & 0.76 & 0.55 \\
\hline & $51-60$ & 0.2 & 1.2 & 0.57 & 0.25 \\
\hline & $61-70$ & 0.2 & 1.9 & 0.64 & 0.33 \\
\hline & $71-80$ & 0.2 & 0.8 & 0.52 & 0.16 \\
\hline & $81-100$ & 0.4 & 1.0 & 0.60 & 0.34 \\
\hline \multirow[t]{6}{*}{ HBA1c } & $30-40$ & 5.9 & 10.0 & 7.59 & 1.21 \\
\hline & $41-50$ & 4.7 & 13.1 & 7.60 & 1.91 \\
\hline & $51-60$ & 5.3 & 13.6 & 8.25 & 2.00 \\
\hline & $61-70$ & 5.4 & 14.5 & 7.90 & 1.90 \\
\hline & $71-80$ & 5.6 & 9.6 & 7.11 & 1.13 \\
\hline & $81-100$ & 6.3 & 7.9 & 7.33 & 0.89 \\
\hline \multirow[t]{6}{*}{ FBS } & $30-40$ & 55.7 & 153.0 & 108.79 & 27.76 \\
\hline & $41-50$ & 78.1 & 267.6 & 132.12 & 50.46 \\
\hline & $51-60$ & 66.3 & 290.0 & 136.02 & 52.93 \\
\hline & $61-70$ & 64.6 & 425.7 & 140.66 & 62.43 \\
\hline & $71-80$ & 77.3 & 270.1 & 126.69 & 51.75 \\
\hline & $81-100$ & 100.0 & 122.7 & 113.23 & 11.80 \\
\hline \multirow[t]{6}{*}{ PPBS } & $30-40$ & 100.0 & 270.0 & 140.35 & 52.92 \\
\hline & $41-50$ & 84.0 & 454.0 & 164.16 & 83.78 \\
\hline & $51-60$ & 100.0 & 431.0 & 184.18 & 73.59 \\
\hline & $61-70$ & 59.0 & 486.0 & 199.74 & 96.69 \\
\hline & $71-80$ & 84.5 & 327.6 & 168.34 & 65.76 \\
\hline & $81-100$ & 124.0 & 329.0 & 233.13 & 103.14 \\
\hline
\end{tabular}


Pearson's correlation was done to evaluate the significance of HbA1c with FBS, PPBS and age of diabetes controlled and poorly controlled patients (Table 04 ). There was a significant positive correlation between HbA1C and FBS $(r=0.0 .369$, p value $<0.001)$ with the $\mathrm{R} \mathrm{Sq}$ of 0.0 .136 and showing a moderate linear relationship (Fig. 1).

Table 04

Pearson's Correlation between $\mathrm{HbA} 1 \mathrm{c}$ and mean results of FBS, PPBS and age

\begin{tabular}{|llll|}
\hline & $\begin{array}{l}\text { Correlation } \\
\text { (r value) }\end{array}$ & R & Squared value \\
\hline Age & -0.010 & 1.019 & 0.887 \\
FBS & 0.369 & 0.136 & 0.000 \\
PPBS & 0.436 & 0.19 & 0.000 \\
\hline
\end{tabular}

A significant positive correlation between HbA1c and PPBS $(r=0.436, p$ value $<0.001)$ showed a moderate linear relationship with $\mathrm{R} \mathrm{Sq}$ of 0.19 . There was no statistically significant relationship between $\mathrm{HbA1c}$ and age.

Our study population was grouped into two according to the HbA1c levels. There were 80 subjects in the well-controlled diabetes group and 121 were included into the poorly controlled group.

The independent sample t-test was done between well- controlled and poorly controlled diabetes groups to compare the mean values of total bilirubin levels, HbA1c, FBS, PPBS and age. (Table 05)

Table 05

Two diabetes groups with variables - independent sample t test ( $p$ value)

\begin{tabular}{|c|c|c|c|c|c|}
\hline Variable & Diabetes Group (HBA1c) & Frequency & Mean & Std. Deviation & $p$ value \\
\hline \multirow[t]{2}{*}{ Total Bilirubin } & $=<7$ & 80 & 0.686 & 0.44 & \multirow[t]{2}{*}{0.001} \\
\hline & $>7$ & 121 & 0.572 & 0.23 & \\
\hline \multirow[t]{2}{*}{ FBS } & $=<7$ & 80 & 113.885 & 26.02 & \multirow[t]{2}{*}{0.000} \\
\hline & $>7$ & 121 & 147.421 & 63.82 & \\
\hline \multirow[t]{2}{*}{ PPBS } & $=<7$ & 80 & 142.862 & 43.73 & \multirow[t]{2}{*}{0.000} \\
\hline & $>7$ & 121 & 209.176 & 92.64 & \\
\hline \multirow[t]{2}{*}{ Age } & $=<7$ & 80 & 59.500 & 11.58 & \multirow[t]{2}{*}{0.119} \\
\hline & $>7$ & 121 & 59.207 & 9.88 & \\
\hline
\end{tabular}


The mean values of total bilirubin, FBS and PPBS of two diabetes groups were compared and it showed statistically significant relationship as shown in the t test ( $p$ value $<0.05$ ). But there was no statistically significant relationship $(p$ value $=0.119)$ between the mean values of age of the two diabetes groups.

Pearson's correlation was done to evaluate the effect of total bilirubin levels on the measurement of HBA1c, FBS and PPBS in all diabetic patients (Table 06). There was a significant negative correlation between total bilirubin and $\mathrm{HbA1c}(r=-0.234$, $p$ value $=0.001)$ which also revealed a low linear relationship ( $\mathrm{R} \mathrm{Sq}$ of 0.055 , Fig. 02 ). There was no statistically significant relationship between the total bilirubin level and FBS, PPBS and age ( $p$ value $>0.05$ ).

\section{Table 06}

Pearson's Correlation between total bilirubin and mean results of $\mathrm{HbA1C}$ FBS, PPBS and age of all diabetic patients.

\begin{tabular}{|llll|}
\hline & $\begin{array}{l}\text { Correlation } \\
\text { (r value) }\end{array}$ & R & S -value \\
\hline Age & -0.103 & 0.011 & 0.147 \\
FBS & -0.107 & 0.011 & 0.131 \\
PPBS & -0.059 & 0.003 & 0.408 \\
HbA1c & -0.234 & 0.055 & 0.001 \\
\hline
\end{tabular}

The mean values of $\mathrm{HbA} 1 \mathrm{c}$ in the male (7.58\%) and female (7.98\%) were compared by independent sample t- test and the $p$ value was 0.965 . There was no statistically significant difference between these variables.

\section{Discussion}

Glycemic control in diabetes mellitus is really important in reducing morbidity and mortality of the disease. Monitoring HbA1c level, FBS and PPBS play a major role in diabetes control. HbA1c level and daily monitoring of plasma blood glucose levels are very important to set up daily blood sugar testing goals to achieve HbA1c levels that having low risk of diabetes outcomes. In resource poor setting, it is difficult to perform $\mathrm{HbA1c}$ and there is no consensus whether only FBS and PPBS are better predictors in diabetes assessment. On other hand $\mathrm{HbA} 1 \mathrm{c}$ also neither captures blood sugar fluctuations in short periods of time nor provide any information on recent control of diabetes. In our research we were able to prove that, there was a statistically significant positive correlation between HbA1c with FBS and PPBS of controlled and poorly controlled diabetic patients.

$\mathrm{HbA1c}$ test is more expensive than the conventional FBS and PPBS estimations requiring sophisticated equipment. However, $\mathrm{HbA} 1 \mathrm{c}$ is an easy test for the patient as it requires a random sample of EDTA blood, unlike FBS and PPBS where the samples have to be collected at the specific periods of time. ${ }^{8}$ 
A study of Haghighatpanah $M$ et al showed that there was a moderate correlation between $\mathrm{HbA} 1 \mathrm{c}$ level and FBS $(r=0.528)$ and PPBS $(r=0.510)$ and this finding were consistent with the findings of Sikaris et $\mathrm{al}^{9}$ and Ketema et $\mathrm{al}^{10}$. In this research, the correlation between PPBS and HbA1c had greater sensitivity, specificity, positive predictive value and negative predictive value compared to the FBS in South Indian T2DM population. ${ }^{11} \mathrm{~A}$ study of Begum $\mathrm{G} S$ et al also showed that both FBS and PPBS correlated significantly with $\mathrm{HbA1c}$ values. PPBS correlated more strongly with $\mathrm{HbA} 1 \mathrm{c}$ in comparison with $\mathrm{FBS} .{ }^{12}$ The findings of our study were also showed more correlation between HbA1c and PPBS $(r=0.436)$ than FBS $(r=0.369)$.

Oxidative stress refers to the imbalance between free radicals and their stabilizing agents, antioxidant enzymes in the body. Obesity, high fatty diets, more sugar consumption, processed foods, exposure to radiation, smoking, alcohol consumption, certain medications and exposure to chemicals are the risk factors of developing oxidative stress. oxidative stress is a harmful process and it negatively affects several cellular structures, such as membranes, lipids, proteins, lipoproteins and deoxyribonucleic acid. ${ }^{13}$

Overproduction of mitochondrial superoxide in endothelial cells of both large and small vessels, and also in the myocardium is a result of metabolic abnormalities of diabetes and causing oxidative stress. This oxidative stress plays an important role in the development of diabetes complications, both microvascular and cardiovascular.

Bilirubin is now considered more than just the final product of heme catabolism. Today it is a fundamental substance that acts as an antioxidant and anti-inflammatory agent in the serum. ${ }^{14}$

A study of Korean T2DM patients showed a significant negative association between total bilirubin and $\mathrm{HbA1c}$, which is independent of sex, age, abdominal circumference, smoking, and other risk factors. ${ }^{15}$

A review study found that low serum bilirubin concentrations are associated with an increased risk of these pathologic conditions such as cardiovascular diseases; whereas, mildly elevated serum bilirubin levels provide protection. ${ }^{16}$

One limitation of this study was, we could not find the information of the compounding factors such as BMI, smoking status, drinking status, exercise habits of the patients.

\section{Conclusion}

We conclude that, there was a statistically significant positive correlation between $\mathrm{HbA} 1 \mathrm{c}$ with FBS and PPBS of diabetes controlled and poorly controlled patients. Statistically significant relationship was observed between the mean values of total bilirubin, FBS and PPBS in the well- controlled and poorly controlled diabetes groups.

There was a significant negative correlation between total bilirubin and $\mathrm{HbA} 1 \mathrm{c}$ in the diabetes patients, indicating the patients with good sugar control (Lower HbA1c) had a higher bilirubin. This would mean 
that a lower blood glucose level will reduce the oxidant stress mediated damage by increasing the oxygen free radicals as a result of the antioxidant and anti- inflammatory action of bilirubin which could result in the reduction of macrovascular manifestations of diabetes. There was no statistically significant difference between the mean values of $\mathrm{HbA} 1 \mathrm{c}$ in the male and female patients.

\section{Abbreviations}

1. Type 2 Diabetes mellitus(T2DM)

2. Glycated Haemoglobin (HbA1c)

3. Fasting blood sugar(FBS)

4. Post prandial blood sugar(PPBS)

5. Advanced glycation end products (AGE)

6. Statistical package for social sciences (SPSS)

\section{Declarations}

- Ethics approval and consent to participate - Was Obtained from,

\section{NAME OF INSTITUTION ETHICAL COMMITTEE -}

Ethical Review Committee Sri Jayewardenepura General Hospital and Postgraduate Training Centre Thalapathpitiya Nugegoda Sri Lanka

\section{ETHICAL REVIEW REFERENCE - (Ref. SJGH/21/ERC/05)}

Study was submitted to and approved by your institutional ethics committee and approval given on $10 / 03 / 2021$. It stated as follows - "We are pleased to inform you that ethical approval has been granted for the above research project which could be effective from the above date of approval"

CONSENT TO PARTICIPATE - The data was obtained from the hospital data base and there was not direct participation of patients and their identity was not revealed in any part of the study. As such the institution ethical committee approved after submission of the project proposal which clearly indicated this.

- Consent for publication-

NOT APPLICABLE

\section{- Availability of data and materials -}

The datasets used and/or analysed during the current study are available from the corresponding author on reasonable request. 
All data generated or analysed during this study are included in this published article [and its supplementary information files].

\section{- Competing interests}

"The authors declare that they have no competing interests"

\section{- Funding - SELF FUNDING}

\section{- Authors' contributions -}

CC Kariyawasan (CK) - Interpretation of data, has drafted the work and substantively revised it and approved the submitted version and have agreed both to be personally accountable for the author's own contributions and to ensure that questions related to the accuracy or integrity of any part of the work.

B L T Balasuriya (TB)- Contributions to the conception and design of the work; the acquisition, analysis, interpretation of data and approved the submitted version and have agreed both to be personally accountable for the author's own contributions and to ensure that questions related to the accuracy or integrity of any part of the work.

S A C D Ranatunga (CR) - Contributions to the conception and design of the work; the acquisition, analysis, interpretation of data and approved the submitted version and have agreed both to be personally accountable for the author's own contributions and to ensure that questions related to the accuracy or integrity of any part of the work.

D M C Dissanayake (CD) - The acquisition, analysis, interpretation of data and approved the submitted version and have agreed both to be personally accountable for the author's own contributions and to ensure that questions related to the accuracy or integrity of any part of the work.

S R G P Herath (GH) - The acquisition, analysis, interpretation of data and approved the submitted version and have agreed both to be personally accountable for the author's own contributions and to ensure that questions related to the accuracy or integrity of any part of the 
work.

- Acknowledgements - NOT APPLICABLE

- Authors' information (optional)

Chitranga Chysanthi Kariyawasan (Corresponding Author) - Consultant Haematologist

Sri Jayewardenepura General Hospital

Balasuriya Lekamlage Thushara Balasuriya - Medical laboratory Technologist (MLT)

Sri Jayewardenepura General Hospital

Sondapperuma Arachchige Charith Dakshitha Ranatunga - Medical laboratory

technologist - Sri Jayewardenepura General Hospital

Dissanayake Mudiyanselage Champika Dissanayake - Medical laboratory technologist

Sri Jayewardenepura General Hospital

Singappuli Ralalage Gemunu Priyantha Herath - Medical laboratory technologist

Sri Jayewardenepura General Hospital

\section{References}

1. American Diabetes A. Diagnosis and classification of diabetes mellitus. Diabetes Care. 2014;37(Suppl 1):81-90.

2. Collaboration NCDRF. Worldwide trends in diabetes since 1980: a pooled analysis of 751 populationbased studies with 4.4 million participants. Lancet. 2016;387:1513-30.

3. Wijewardene K, Mohideen MR, Mendis S, Fernando DS, Kulathilaka T, et al. Prevalence of hypertension, diabetes and obesity: baseline findings of a population based survey in four provinces in Sri Lanka. Ceylon Med J. 2005;50:62-70.

4. Ghazanfari Z, Haghdoost AA, Alizadeh SM, Atapour J, Zolala F. A comparison of HbA1c and fasting blood sugar tests in general population. Int J PrevMed. 2010;1(3):187-94.

5. Sultanpur CM, Deepa K, Kumar SV. Comprehensive review on HbA1c in diagnosis of diabetes mellitus, International Journal of Pharmaceutical Sciences Review and Research,2010:3:119-122.

6. UK Prospective Diabetes Study (UKPDS) Group. Intensive blood-glucose control with sulphonylureas or insulin compared with conventional treatment and risk of complications in patients with type 2 diabetes (UKPDS 33). Lancet. 1998;352:837-53. 
7. Erkus $E^{\star}$, Aktas G, Kocak MZ, Duman TT, Atak BM. Serum bilirubin level is associated with diabetic control in type 2 diabetes mellitus, Blood, Heart and Circulation,2018: Volume 2(2): 1-2.

8. Vani K, Renuka A. Correlation of glycated haemoglobin with fasting and post prandial blood glucose in Type 2 diabetes. International Journal of Clinical Biochemistry Research. 2020;7(3):380-3.

9. Sikaris K. The correlation of hemoglobin A1c to blood glucose. J Diabetes Sci Technol. 2009;3:42938.

10. Ketema EB, Kibret KT. Correlation of fasting and postprandial plasma glucose with HbA1c in assessing glycemic control; systematic review and meta-analysis. Arch Public Health. 2015;73:1-9.

11. Haghighatpanah M, Thunga G, Khare S, Mallayasamy S. Correlation of glycosylated hemoglobin levels with fasting and postprandial glucose in south indian type 2 diabetic patients, International Journal of Pharmacy and Pharmaceutical Sciences.2016:8(8): 285-288.

12. Begum GS, Jabeen A, Rahman A. Assessing The Glycemic Control in Type 2 Diabetic Patients By Comparing Glycosylated Hemoglobin Levels (Hba1c) With Fasting And Postprandial Glucose, Journal of Biotechnology and Biochemistry.2018:4(1):54-57.

13. Manisha HW, Rajak R, Jat D. Oxidative stress and antioxidants: an overview, International Journal of Advanced Research and Review.2017:2(9): 110-119.

14. Regino WO, Velasco H, Sandoval $\mathrm{H}$. The protective role of bilirubin in human beings, Rev Col Gastroentero,2009:24(3):287.

15. Choi S, Lee Y, Kwen S, Song H, Ahn H, Rhee J, Choi J, Shin M. Association between Total Bilirubin and Hemoglobin A1c in Korean Type 2 Diabetic Patients. J Korean Med Sci. 2012;27:1196-201.

16. Vitek L. The role of bilirubin in diabetes, metabolic syndrome, and cardiovascular diseases, Frontiers in Pharmacology | Drug Metabolism and Transport.2012:3(55):1-7.

\section{Figures}



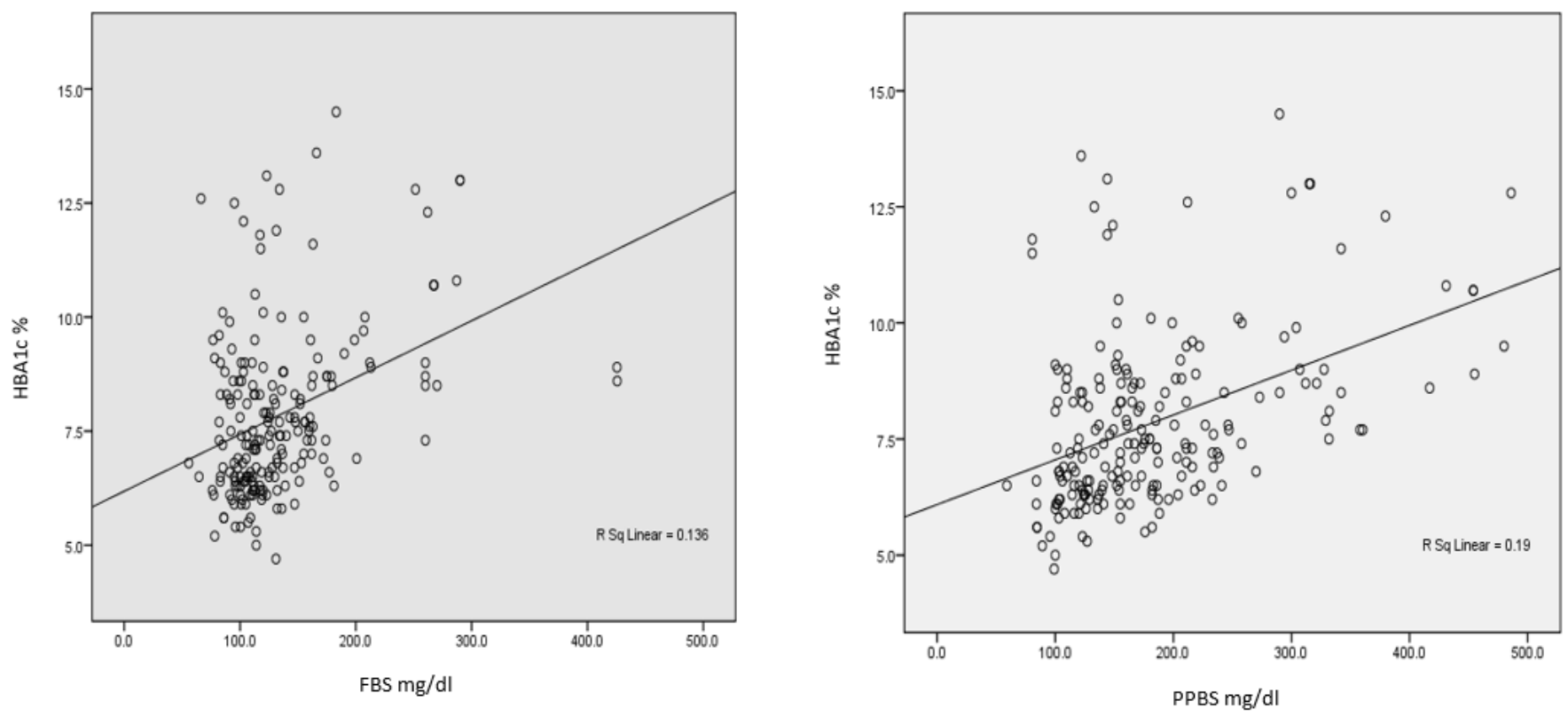

Figure 1

Scatter diagram of FBS and PPBS with HBA1C

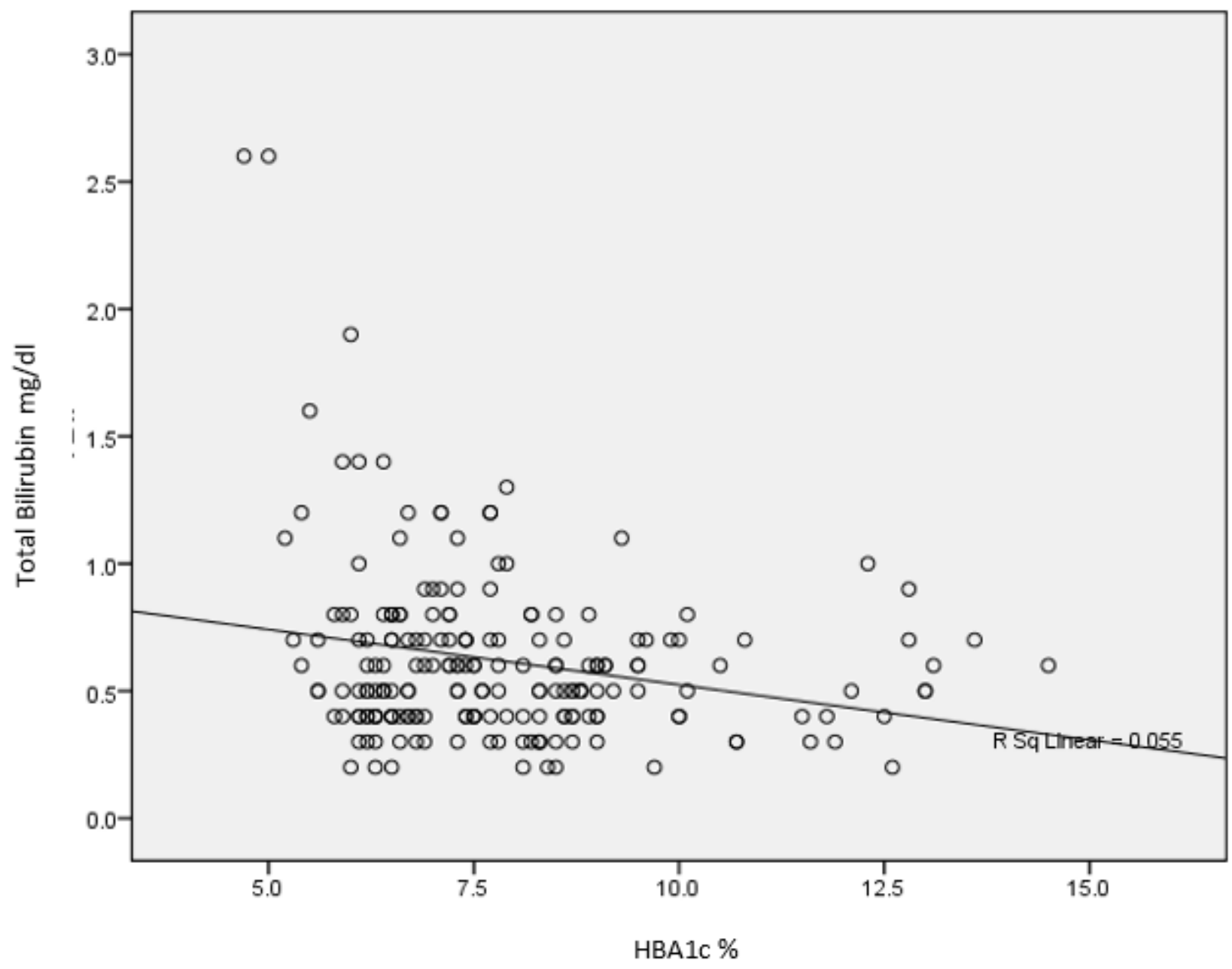

Page 14/15 
Figure 2

Scatter diagram of HBA1c with Total bilirubin 